\title{
Correlated Production and Analog Transport of Fission Neutrons and Photons using Fission Models FREYA, FIFRELIN and the Monte Carlo Code TRIPOLI- $4^{\circledR}$.
}

\author{
Jérôme M. Verbeke \\ Odile Petit \\ Lawrence Livermore National Laboratory Commissariat à l'Energie Atomique \\ Nuclear and Chemical Sciences Division \\ P.O. Box 808 \\ Livermore, California 94551-0808 \\ U.S.A. \\ Email: Verbeke2@1lnl.gov \\ et aux Energies Alternatives \\ Université Paris-Saclay \\ F-91191 Gif-sur-Yvette, cedex \\ France \\ Email: Odile.Petit@cea.fr
}

\author{
Olivier Litaize \& Abdelhazize Chebboubi \\ Commissariat à l'Energie Atomique \\ et aux Energies Alternatives \\ F-13108 Saint-Paul-lès-Durance \\ France \\ Email: Olivier.Litaize@cea.fr \\ Abdelhazize.Chebboubi@cea.fr
}

\begin{abstract}
Fission modeling in general-purpose Monte Carlo transport codes often relies on average nuclear data provided by international evaluation libraries. As such, only average fission multiplicities are available and correlations between fission neutrons and photons are missing. Whereas uncorrelated fission physics is usually sufficient for standard reactor core and radiation shielding calculations, correlated fission secondaries are required for specialized nuclear instrumentation and detector modeling. For coincidence counting detector optimization for instance, precise simulation of fission neutrons and photons that remain correlated in time from birth to detection is essential. New developments were recently integrated into the Monte Carlo transport code TRIPOLI-4 to model fission physics more precisely, the purpose being to access event-by-event fission events from two different fission models: FREYA and FIFRELIN. TRIPOLI-4 simulations can now be performed, either by connecting via an API to the LLNL fission library including FREYA, or by reading external fission event data files produced by FIFRELIN beforehand. These new capabilities enable us to easily compare results from Monte Carlo transport calculations using the two fission models in a nuclear instrumentation application. In the first part of this paper, broad underlying principles of the two fission models are recalled. We then present experimental measurements of neutron angular correlations for ${ }^{252} \mathbf{C f}(\mathbf{s f})$ and ${ }^{240} \mathrm{Pu}(\mathbf{s f})$. The correlations were measured for several neutron kinetic energy thresholds. In the latter part of the paper, simulation results are compared to experimental data. Spontaneous fissions in ${ }^{252} \mathrm{Cf}$ and ${ }^{240} \mathrm{Pu}$ are modeled by FREYA or FIFRELIN. Emitted neutrons and photons are subsequently transported to an array of scintillators by TRIPOLI-4 in analog mode to preserve their correlations. Angular correlations between fission neutrons obtained independently from these TRIPOLI-4 simulations, using either FREYA or FIFRELIN, are compared to experimental results. For ${ }^{240} \mathrm{Pu}(\mathrm{sf})$, the measured correlations were used to tune the model parameters.
\end{abstract}

\section{INTRODUCTION}

Spontaneous fission is characterized by the emission of bursts of neutrons. If multiplying fissile materials surround these spontaneous fissions, the bursts are amplified to form fission chains. This unique fission chain signature has been used for many decades to detect and authenticate nuclear materials. Most nonproliferation and verification neutron measurement systems rely on detecting thermalized neutrons. Typically ${ }^{3} \mathrm{He}$ tubes record the arrival times of thermalized neutrons. Unfortunately, the neutron properties at the time of emission are obscured by down-scattering. Scintillators, on the other hand, directly detect unmoderated fission neutrons because inelastic scattering of neutrons on hydrogen results in the emission of a recoil proton, ionizing the scintillation material, enabling detection on a nanosecond time scale. When fast neutron detectors are used, the neutron-neutron correlation from fission can be used to characterize fissile samples.

With fast detectors, characterization of nuclear materials becomes possible by measurements of the fission neutron angular anisotropy. In spontaneous and low energy fission, almost all of the neutron emission comes from the fully accelerated fission fragments whose back-to-back motion is imprinted on the neutron directions in the laboratory frame. Thus small angle correlations are expected from neutrons emitted from the same fragment, whereas large angle correlations arise from opposite fragments. Anisotropy measurements can provide valuable information for identifying the composition of materials.

General-purpose Monte Carlo codes are available for modeling neutron transport. They have traditionally employed "average fission models" for modeling fission, characterized by uncorrelated secondary particle emission. This approximation is sufficient for the calculation of average quantities such as flux, energy deposition and multiplication. However, correlations are important for modeling anisotropy of fission neutrons, because measurement of anisotropy relies on measuring timecorrelated neutrons.

Based on physics models and experimental data, the eventby-event fission generators FREYA and FIFRELIN are able to predict a host of correlation observables, including correlations 
in neutron multiplicity, energy, and angles, and the energy sharing between neutrons and photons. The combination of the Monte Carlo radiation transport code TRIPOLI-4.10 with these fission generators enables users to directly model fission eventby-event and transport fission secondaries through complex detector geometries while keeping them fully correlated from birth to detection.

The paper is organized as follows. We first describe the fission event generators FREYA, FIFRELIN and the Monte Carlo radiation transport code TRIPOLI-4. We then present experimental measurements of neutron-neutron angular correlations between ${ }^{252} \mathrm{Cf}(\mathrm{sf})$ and ${ }^{240} \mathrm{Pu}$ (sf) fission neutrons. Next, we compare these correlations to those obtained by simulations and discuss the differences between the two fission models. Model parameters are adjusted to best match the experimental data.

\section{FISSION EVENT GENERATORS}

\section{A. FREYA}

FREYA [1]-[9] requires the fission fragment mass distribution $\mathrm{Y}(\mathrm{A})$ and the total kinetic energy $\mathrm{TKE}(\mathrm{A})$ for the particular excitation considered (FREYA can simulate both neutroninduced fission and spontaneous fission). The yields $\mathrm{Y}(\mathrm{A})$ are either taken to be the measured fragment yields directly [3] or a five-Gaussian fit to the data which makes it possible to parameterize the energy dependence of the yields [4] in the case of neutron-induced fission. In order to generate an event, FREYA first selects the mass split based on the provided $\mathrm{Y}(\mathrm{A})$. The fragment charges are then sampled from normal distributions. The linear and angular momenta of the two fragments and their internal excitations are subsequently sampled, as explained below. After their formation, the fully accelerated fragments de-excite first by neutron evaporation and then by photon emission. For a given split of nucleus $\mathrm{A}_{0}$ into light and heavy fragments, $\mathrm{L}$ and $\mathrm{H}$ respectively, the Qvalue is given by $Q=M_{0} c^{2}-M_{L} c^{2}-M_{H} c^{2}$. For a given total fragment kinetic energy TKE, the total excitation energy available for rotational and statistical excitation of the two fragments is then $E_{s c}^{*}=Q-\overline{T K E}$ and the corresponding 'scission temperature' $T_{s c}$ is obtained from $E_{s c}^{*}=\left(A_{0} / e_{0}\right) T_{s c}^{2}$ where $e_{0}$ is the scale of the level density parameter. The inclusion of angular momentum in FREYA was described in Ref. [6]. In addition to any overall rigid rotation of the dinuclear configuration prior to scission, which imparts a mean angular momenta to the two fragments, the fragments also acquire fluctuations around those mean values from the wriggling and bending modes (consisting of rotations in the same or opposite sense around an axis perpendicular to the dinuclear axis) but ignores tilting and twisting modes (in which the fragments rotate around the dinuclear axis). The degree of these fluctuations is governed by the adjustable parameter $c_{S}: T_{S}=c_{S} T_{s c}$. The fluctuations vanish for $c_{S}=0$ so the fragments emerge with the angular momenta dictated by the overall rigid rotation of the scission configuration (which is usually very small for induced fission - and entirely absent for spontaneous fission). The default value, $c_{S}=1$, leads to
$\bar{S}_{L} \sim 6.2 \hbar$ and $S_{H} \sim 7.6 \hbar$ for ${ }^{252} \mathrm{Cf}(\mathrm{sf})$, see Ref. [6] for details.

After accounting for the total rotational energy of the two fragments, $E_{r o t}$, one is left with a total of $E_{s t a t}=E_{s c}^{*}-E_{\text {rot }}$ for statistical fragment excitation that is distributed between the two fragments.

First a preliminary partition, $E_{\text {stat }}=\dot{E}_{L}^{*}+\dot{E}_{H}^{*}$, is made according to the heat capacities of the two fragments which are assumed to be proportional to the corresponding Fermigas level density parameters, i.e. $\dot{E}_{L} / \dot{E}_{H}=a_{L} / a_{H}$, where the level density parameter $a$ can depend on the pairing energy and shell corrections of the fragment. If the shell corrections are negligible, or the available energy is large, then $a_{i}=A_{i} / e_{0}$, i.e. $a_{i}$ is simply proportional to the fragment mass number $A_{i}$, and this renormalization is immaterial. A default value of $e_{0}=10 / \mathrm{MeV}$ is typically used. If the two fragments are in mutual thermal equilibrium, $T_{L}=T_{H}$, the total excitation energy will, on average, be partitioned as above. But because the observed neutron multiplicities suggest that the light fragments tend to be disproportionately excited, the average excitations are modified in favor of the light fragment,

$$
\bar{E}_{L}^{*}=x \dot{E}_{L}^{*}, \bar{E}_{H}^{*}=E_{\text {stat }}-\bar{E}_{L}^{*},
$$

where the adjustable model parameter $x$ is expected be larger than unity. It was found that $x=1.27$ leads to reasonable agreement with $\nu(A)$ for ${ }^{252} \mathrm{Cf}(\mathrm{sf})$ [3].

After the mean fragment excitation energies have been assigned, FREYA considers the effect of thermal fluctuations. In Weisskopf's statistical model of the nucleus, which describes the excited nucleus as a degenerate Fermi gas, the mean excitation of a fragment is related to its temperature $T_{i}$ by $\bar{E}_{i}^{*}=\tilde{a}_{i} T_{i}^{2}$ [10]-[12] and the associated variance in the excitation is $\sigma_{E_{i}}^{2}=-\partial^{2} \ln \rho_{i}\left(E_{i}\right) / \partial E_{i}^{2}=2 \bar{E}_{i}^{*} T_{i}$. Therefore, for each of the two fragments, an energy fluctuation $\delta E_{i}^{*}$ is sampled from a normal distribution of variance $2 c^{2} \bar{E}_{i}^{*} T_{i}$. The fragment excitations are adjusted accordingly with

$$
E_{i}^{*}=\bar{E}_{i}^{*}+\delta E_{i}^{*}, i=L, H .
$$

Energy conservation is accounted for by making a compensating opposing fluctuation in the total kinetic energy,

$$
\mathrm{TKE}=\overline{\mathrm{TKE}}-\delta E_{L}^{*}-\delta E_{H}^{*} .
$$

The average TKE, TKE, has been adjusted by $d$ TKE to reproduce the average neutron multiplicity, $\bar{\nu}$. The factor $c$ multiplying the variance explores the effect of the truncation of the normal distribution at the maximum available excitation. Its value affects the neutron multiplicity distribution $P(\nu)$. A default value, $c=1.0$, had previously been assumed.

The evaporated neutrons are assumed to be isotropic in the frame of the emitting nucleus, apart from a very slight flattening due to the nuclear rotation. Their energy is sampled from a black-body energy spectrum, $d N_{n} / d E_{n} \sim$ $E_{n} \exp \left(-E_{n} / T_{\max }\right)$, where $T_{\max }$ is the maximum possible temperature in the daughter nucleus (corresponding to emission of a very soft neutron). 
FREYA generally assumes that neutron evaporation continues until the nuclear excitation energy is below the threshold $S_{n}+Q_{\min }$, where $S_{n}$ is the neutron separation energy and $Q_{\min }$ is small. The default value is $Q_{\min }=0.01 \mathrm{MeV}$, so that neutron evaporation continues as long as energetically possible. After neutron evaporation has ceased, the excited product nucleus will undergo sequential photon emission to rid itself of the remaining excitation energy. First, statistical photons are emitted isotropically with an energy distribution sampled from a black-body spectrum modulated by a giantdipole resonance form factor. Each emission reduces the magnitude of the angular momentum by $d S=1 \hbar$. The cascade is continued until the statistical excitation is below a specified threshold, $\epsilon_{m i n}$, which can be detector dependent. However, when the photon decay cascade leads to an excitation below any of the tabulated RIPL-3 [13] levels, FREYA switches to a discrete cascade based on the RIPL-3 data. The discrete cascade is continued until the half-life $t_{\ell}$ exceeds a specified value, $t_{\max }$, which should be adjusted to reflect the detector response time. If the photon decay for a particular fragment is not in the RIPL-3 tables and the statistical excitation is below $\epsilon_{\text {min }}$, the remaining de-excitation occurs by collective photon emission that each reduce the angular momentum by $2 \hbar$.

\section{B. FIFRELIN}

A FIFRELIN calculation consists on one hand in sampling the initial state of a pair of fission fragments emerging from a binary fission event (mass A, nuclear charge $\mathrm{Z}$, excitation energy $\mathrm{E}$, spin $\mathrm{J}$ and parity $\pi$ ) and on the other hand in deexciting both fragments [14]-[17]. In this work, mass and kinetic energy are sampled from experimental data [18], [19] while nuclear charge is estimated from unchanged charge density hypothesis, corrected by polarization function and proton/neutron even-odd factors. Total excitation energy is derived from energy conservation since kinetic energy has already been sampled from experimental data.

A rotational energy (collective mode of excitation) is calculated from a simple rotating liquid drop model involving a nuclear moment of inertia and a total angular momentum which is sampled following Bethe's work [20]: $P(J)=(J+$ $1 / 2) / \sigma^{2} \exp \left(-(\mathrm{J}+1 / 2)^{2} / 2 \sigma^{2}\right)$. For primary fission fragments (before de-excitation), the spin cut-off parameter $\sigma$ is a free parameter (one for light fragment group $\sigma_{L}$ and another one for heavy group $\sigma_{H}$ ). A third free parameter is the fraction of rigid body moment of inertia involved in the calculation of the rotational energy but it is generally fixed to one.

Intrinsic excitation energy (obtained after subtracting rotational energy from total excitation energy) is shared between fragments using an $E=a T^{2}$ relation involving the level density parameter $a$ (following Ignatyuk's prescription) and the temperature $T$ of the fragment. This system of two equations (one equation per fragment) is solved by iteration since the level density parameter is energy dependent and the excitation energy is the unknown quantity.

For a given fragmentation, the temperature of the light fragment $T_{L}$ is linked to the temperature of its heavy partner $T_{H}$ by a mass dependent temperature ratio law $R_{T}(A)=T_{L} / T_{H}$. This law involves two additional free parameters $R_{T}^{\min }, R_{T}^{\max }$.

Finally five free parameters have been described to simulate the entry zone in energy/spin of the primary fission fragments. These parameters will be chosen to reproduce a target fission observable such as average prompt neutron multiplicity $\bar{\nu}(3.76$ for ${ }^{252} \mathrm{Cf}$ ).

The de-excitation process in an energy, spin, parity set can be solved through the so-called Hauser-Feshbach formalism accounting for neuton/gamma competition. In FIFRELIN, it has been implemented in a specific way using the notion of Nuclear Realization developed in [21] for gamma emission and extended here to neutron/gamma coupled emission [15], [16]. A nuclear realization is a nuclear level scheme (energy $E$, spin $J$, parity $\pi$ of a set of levels written $[E J \pi]$ ) plus the associated partial widths $\Gamma_{p}\left([E J \pi]_{i} \rightarrow[E J \pi]_{f}, \alpha\right)$, describing transitions between initial level $i$ and final level $f$. [EJ $]$ can be a unique level or a set of levels inside an energy bin. Parameter $p$ stands for 'particle' and can be a gamma, neutron or conversion electron. Parameter $\alpha$ is related to quantum numbers ( $X L$ for multipolarity-type of gammas and $(l, j)$ for orbital and total angular momenta of neutrons). The algorithm also accounts for Porter-Thomas fluctuations of partial widths during the cascade.

Inside a nuclear level scheme the excitation energy range is decomposed in three parts:

- from the ground state to a cut-off energy $E_{c u t-o f f}$ provided by RIPL-3 database, the level scheme is supposed to be complete and $[E, J, \pi]$ set as well as experimental transition intensities are accounted for (strictly speaking spins are not always known and the code then completes this lack of information by using spin distribution models),

- from $E_{c u t-o f f}$ to $E_{b i n}$ the scheme is completed by theoretical discrete levels sampled from level density models (several models are available in FIFRELIN as well as for photon strength functions involved in the calculation of partial radiative widths).

- from $E_{b i n}$ to the maximum excitation energy available the level scheme is simply decomposed on energy bins (roughly $\delta E=10 \mathrm{keV}$ width). Again theoretical level density models are used to calculate the average number of levels $N\left([E, J, \pi]_{i}\right)$ inside bin $i$.

Finally a standard Monte Carlo procedure is used to sample the different transitions of a cascade that can correspond to neutron, gamma or conversion electron (since internal conversion coefficients are accounted for at low energy).

The simulation of a given cascade is finished when a $\beta^{-}$, $\beta^{-} n$ or ground-state level is reached. In order to compare with fission experiments, a time coincidence window (between fission fragment and gammas) is considered by accounting for half-lives of nuclear levels provided by RIPL-3 database. The emission occurs regarding the probability associated to the half-life of the initial level. A cascade ends when the cumulative time from the beginning of the cascade exceeds the coincidence time-window. 


\section{RADiATION TRANSPORT MONTE CARLO CODE}

TRIPOLI-4 is a continuous-energy Monte Carlo transport code developed in CEA Saclay, France [22]. It simulates neutron, photon, positron and electron transport. It is dedicated to shielding, reactor physics with or without depletion, criticality safety and nuclear instrumentation. Particle histories are simulated in a three-dimensional geometry, either surface-based and/or combinatorial, or produced by the ROOT software. International nuclear data libraries are used in the ENDF/B format to directly access continuous-energy cross sections, emission spectra and anisotropy data. Various variance reduction techniques are also available, and specifically a builtin module based on importance sampling. The TRIPOLI-4 code can be run in parallel on multi-core machines as well as on heterogeneous networks of stations or massive parallel machines.

Previous work has been performed in order to use either FREYA or FIFRELIN fission models, instead of nuclear evaluated data from international libraries, when sampling neutrons emitted by neutron induced fission in TRIPOLI-4. First, the LLNL fission Library was coupled to TRIPOLI-4 to model fission on an event-by-event basis through a direct call to FREYA at each fission [23]. Typically, this led to an increase in simulation time of the order of $15 \%$ only. Then, FIFRELIN fission model and TRIPOLI-4 have been coupled through external files [24]: the standalone FIFRELIN code has to be run beforehand to provide files gathering data for many independent fission events. For both fission models, TRIPOLI4 produces neutrons from these fission events and simulates their transport up to the detectors. Usual Monte Carlo average tallies, such as fluxes or reaction rates, can then be estimated. For nuclear instrumentation applications, it is useful to store TRIPOLI-4 generated particle tracks into ROOT trees, which can easily be post-processed afterwards by imposing cuts on detector variables, e.g. time and position.

Analog transport mode is also available in TRIPOLI-4 for neutrons and photons [25]. It deactivates implicit capture and produces as many individual secondaries as required by the multiplicity of the reaction, for example in the case of neutron scattering with multiple outgoing neutrons.

For this paper, the production of photons in TRIPOLI-4 through the FREYA and FIFRELIN fission models has been added. The principle is the same as for neutrons: photons are produced via the fission model and TRIPOLI-4 transports them. The case of spontaneous fission has also been addressed: for FREYA through an external source linked to TRIPOLI4 which performs itself the calls to FREYA; for FIFRELIN through a special kind of source feature reading neutrons and photons data from the FIFRELIN fission event files.

\section{NEUtRon-NEUtRon ANGULAR CORRELATIONS}

\section{A. Experiments}

Figure 1 shows the geometrical configuration of the detector array used to measure the neutron-neutron correlations. The array consists of seventy-seven scintillators. Each detector in the array is cylindrical in shape, $10.16 \mathrm{~cm}$ diameter by $7.62 \mathrm{~cm}$ deep and filled with EJ-301 scintillating material [26]. Thirteen detectors sit over a cavity formed by an octagonal array underneath. Each arm of the octagon is a vertical tower made of eight scintillators. The measurement cavity is also octagonal, with $60 \mathrm{~cm}$ between the faces of opposite towers, and stands $50 \mathrm{~cm}$ tall. The tightly-packed system has $2 \pi$ solid angle coverage, resulting in an overall geometric efficiency of $50 \%$. Each of the 77 scintillators is individually read out by a photomultiplier tube (PMT).

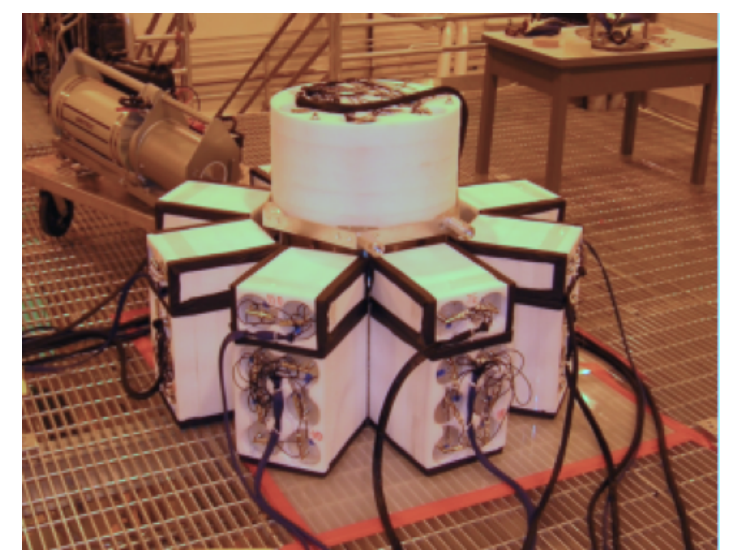

Fig. 1. Photograph of the 77 liquid scintillator array on low mass floor.

All detectors were calibrated in energy using a ${ }^{137} \mathrm{Cs}$ source. Neutron-photon discrimination was achieved using the pulse shape discrimination method, i.e. by simultaneously measuring the charges in the peak and the tail of the PMT pulse. The detectors were synchronized using Compton scattering between neighbors.

The purpose of our measurements is to answer the question "are neutrons from spontaneous fission more likely to be emitted back-to-back, at $90^{\circ}$ or in the same direction?" Correlations between neutrons arise from fissions simultaneously emitting multiple neutrons. In our experimental method, a pair is tagged as correlated when two neutrons are detected within $40 \mathrm{~ns}$ of each other, in which case they are assumed to have originated from the same spontaneous fission. Two detected neutrons are uncorrelated if the second one comes after a delay of at least $100 \mu \mathrm{s}$. A time window opens after that delay to count uncorrelated neutrons. The length of this time window depends on the neutron source strength. It is $1 \mathrm{~ms}$ for our ${ }^{252} \mathrm{Cf}$ (sf) source and $100 \mathrm{~ms}$ for the weaker ${ }^{240} \mathrm{Pu}$ (sf) source. The ratio of correlated to uncorrelated event rates is proportional to the probability of detecting two neutrons from the same spontaneous fission. Since we know the locations of all detectors with respect to the source, we can easily derive this probability as a function of the angular separation between the two neutrons.

The angular correlation between fission neutrons is measured for several neutron kinetic energy cutoffs. Neutron time of flight is applied to filter neutrons in kinetic energy. A photon from spontaneous fission is used to trigger the time-of-flight 
measurement window and a neutron is employed to close it. This time of flight method produces correlation distributions between neutrons that are detector independent. More details on the experimental setup are available in Ref. [27].

Measuring a $230 \mu \mathrm{Ci}{ }^{252} \mathrm{Cf}$ source in the center of the detection system for 30 minutes, we obtained the two-neutron angular correlations shown as full circles in Fig. 2. In this paper, data always refers to the experimental measurements. The different colors identify the neutron kinetic energy cutoffs applied to the neutrons.

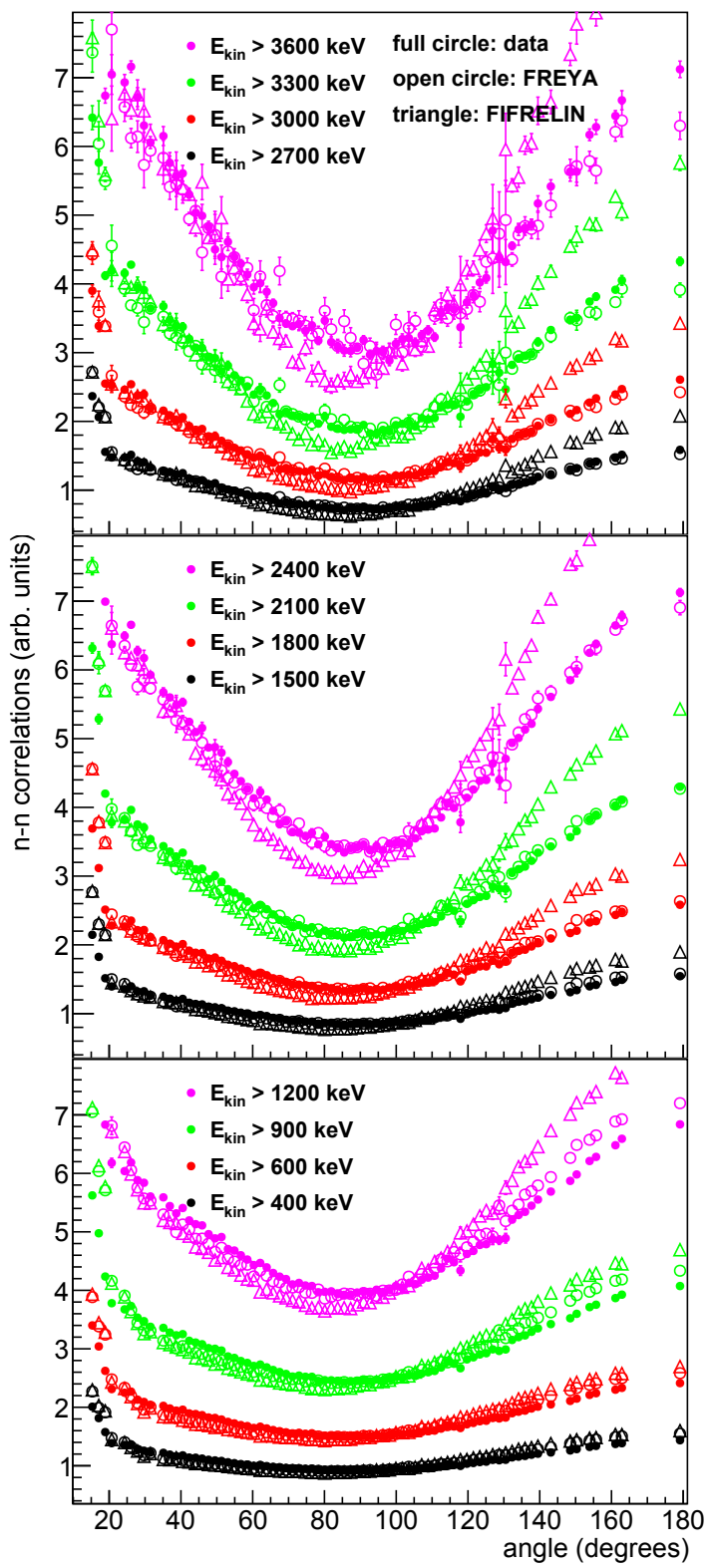

Fig. 2. The cross talk corrected two-neutron angular correlation for ${ }^{252} \mathrm{Cf}(\mathrm{sf})$ as a function of the angular separation for several neutron kinetic energy thresholds.

While not shown here, there is an overall good agreement between our data and other data sets [28], [29], which is encouraging in the light of the fact that our approach is completely independent, including different detectors, experimental setups, and analyses.

All distributions presented in this paper are corrected for neutron cross talk. Cross talk occurs when the same neutron is detected by two or more detectors. It affects primarily the pairs of detectors at the smallest separation angles, because these detectors are neighbors and thus tightly coupled, and they have the least material between them to prevent a neutron from scattering from one detector to the other. There is no reliable experimental analysis that can isolate counts due to cross talk on an event-by-event basis, so we resorted to Monte Carlo simulations using FREYA to remove integral cross talk counts from the experimental coincidences [27]. The simulated cross talk contribution is shown in Fig. 3 as a function of the detector separation angle, as seen from the source. In the simulations, it is important to account for the scintillation light-dependent neutron detection efficiency of the detectors.

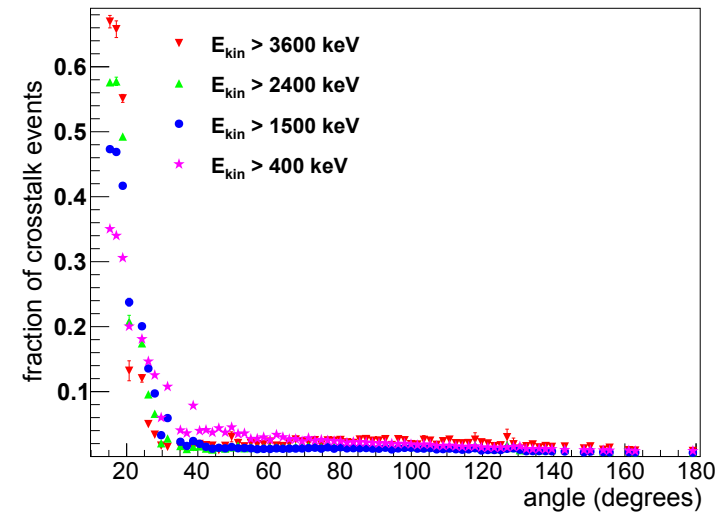

Fig. 3. Fraction of coincidences attributed to neutron cross talk as a function of the detector separation angle and for several neutron kinetic energy thresholds.

Figure 2 shows that for angles smaller than $25^{\circ}$, the neutron cross talk correction appears to undercorrect the correlations, leading to a small rise. Since neutron cross talk correction is estimated by simulations, this rise can be attributed to either inaccurate modeling of either the scintillation material or the scintillator geometry. It could also be attributed to an underprediction of the zero degree correlation in the FREYA simulation of the neutron cross talk.

We then proceeded to measure a 4.5 gm sample of ${ }^{240} \mathrm{Pu}$ ( $98 \%$ pure) of intensity 4,590 neutrons/s. The data analyzed were collected by placing the ${ }^{240} \mathrm{Pu}$ source in the center of the detection system for 23 hours. The two-neutron angular correlations are shown as full circles in Fig. 4.

\section{B. Simulations}

Using TRIPOLI-4 in combination with the fission event generators FREYA and FIFRELIN, we simulated neutronneutron angular correlations.

The first simulations are shown for the ${ }^{252} \mathrm{Cf}(\mathrm{sf})$ source in Fig. 2, where the open circles and triangles are the FREYA and FIFRELIN calculations, respectively. For FREYA and 


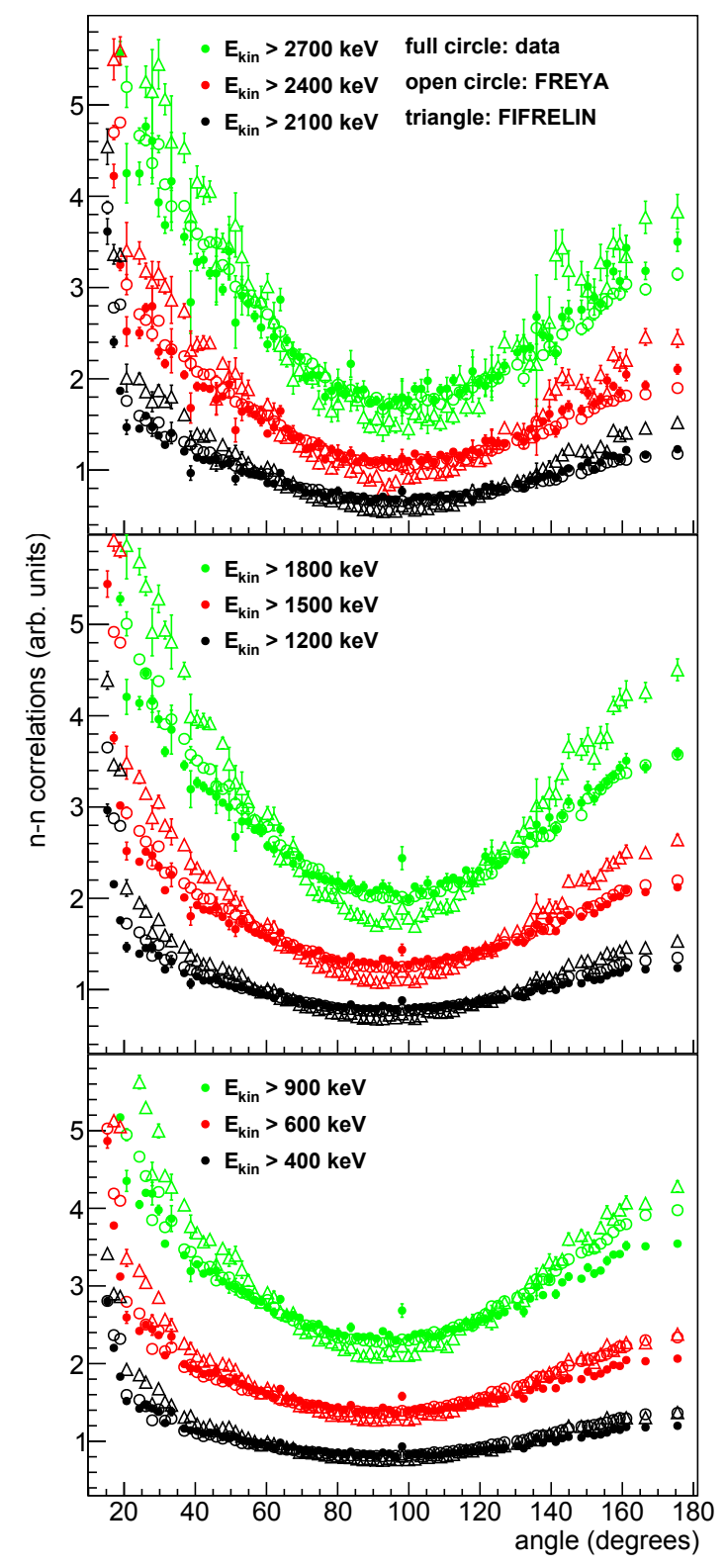

Fig. 4. The cross talk corrected two-neutron angular correlation for ${ }^{240} \mathrm{Pu}(\mathrm{sf})$ as a function of the angular separation and for several neutron kinetic energy thresholds.

FIFRELIN, the numbers of fissions simulated were equivalent to 40 and 56 seconds of data taking, respectively.

The neutron-neutron correlation arises because, while the neutrons are emitted isotropically in the rest frame of the fragment, the boost to the laboratory frame means that the neutrons will preferentially follow the fragments. Thus if one neutron is emitted from each fragment, they will be found at $180^{\circ}$ apart while, if both are emitted from the same fragment, the angular separation is $0^{\circ}$. The $0^{\circ}$ correlation includes two parts: both neutrons emitted from the light fragment and both emitted from the heavy fragment. Since the light fragment is higher velocity to conserve momentum, the correlation from two-neutron emission from the light fragment at $0^{\circ}$ is larger.
The three contributions combine to give peaks at $0^{\circ}$ and $180^{\circ}$ with a dip at $90^{\circ}$.

The light fission fragment also emits neutrons with larger kinetic energy on average, because it receives a disproportionately higher excitation energy than the heavy fragment. Therefore, increasing the neutron energy threshold increases the average energy of the neutrons that remain to form the correlation. Thus the higher the neutron energy threshold, the larger the bias toward emission from the light fragment and the higher the $0^{\circ}$ correlation relative to the back-toback correlation at $180^{\circ}$. In addition, the higher energy cutoff preferentially selects neutrons that are emitted in the direction of the boost rather than those opposite the boost direction which enhances the $0^{\circ}$ correlation as the energy increases.

The simulations for ${ }^{240} \mathrm{Pu}(\mathrm{sf})$ are shown next in Figure 4 with the same open symbols. The numbers of fissions simulated were equivalent to $6 \mathrm{~h}$ and $45 \mathrm{~min}$ of data taking for FREYA and FIFRELIN, respectively.

We note qualitative differences in the ${ }^{252} \mathrm{Cf}(\mathrm{sf})$ and ${ }^{240} \mathrm{Pu}$ (sf) correlations due to the different average neutron multiplicities. Since the average neutron multiplicity of ${ }^{252} \mathrm{Cf}(\mathrm{sf})$ is $\sim 3.76$, each fragment can emit more than one neutron and any two emitted neutrons can be used to form the correlation function. On the other hand, the average neutron multiplicity of ${ }^{240} \mathrm{Pu}(\mathrm{sf})$ is $\sim 2.1$ so that, on average, the neutron-neutron correlation is formed from the only neutrons emitted during the fission. In addition, the average neutron energy of neutrons emitted from ${ }^{252} \mathrm{Cf}(\mathrm{sf})$ is higher than those from ${ }^{240} \mathrm{Pu}(\mathrm{sf})$ so that increasing the energy threshold is more likely to result in two peaks of equal strength for ${ }^{252} \mathrm{Cf}$ (sf) than for ${ }^{240} \mathrm{Pu}(\mathrm{sf})$. These characteristics can be observed in the data as well as in the simulations.

While the simulations do not reproduce the data perfectly, both fission event generators qualitatively reproduce the shapes of the experimental data. Without them the distributions would be flat. For angles smaller than $25^{\circ}$, we generally observe deviations between measurements and simulations, likely due to the reasons stated earlier, i.e. insufficient details for cross talk modeling in the simulation, etc.

Let us discuss the FREYA results. FREYA includes several physics-motivated model parameters. In particular, the parameter $x$ describing how the excitation energy is partitioned between the light and heavy fission fragments. If the two fragments are in mutual thermal equilibrium, the total excitation energy will, on average, be partitioned according to the heat capacities of the fragments. However, observed neutron multiplicities suggest that the light fragment tends to be disproportionately excited. Therefore the average excitation energy is modified in favor of the light fragment. For ${ }^{252} \mathrm{Cf}(\mathrm{sf})$, $x=1.27$, which means that the excitation energy of the light fragment is $\sim 30 \%$ higher than that of the light fragment. For ${ }^{240} \mathrm{Pu}(\mathrm{sf})$, a default value of $x=1.2$ was assumed previously since this was close to the value obtained for ${ }^{239} \mathrm{Pu}\left(n_{\mathrm{th}}, \mathrm{f}\right)$. We note, however, that calculations in Refs. [5], [27], showed that the neutron-neutron angular correlations are sensitive to the value of $x$. Therefore, we could use our data to determine $x$ 
for ${ }^{240} \mathrm{Pu}(\mathrm{sf})$.

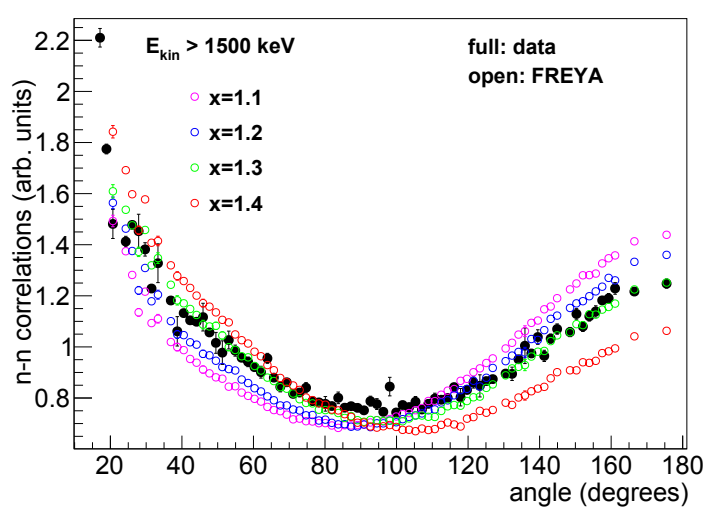

Fig. 5. The cross talk corrected two-neutron angular correlation for ${ }^{240} \mathrm{Pu}(\mathrm{sf})$ as a function of the angular separation and for a neutron kinetic energy threshold of $1500 \mathrm{keV}$. FREYA calculations with $x=1.1,1.2,1.3$ and 1.4 are also shown.

To try to determine the value of $x$ that agrees best with our ${ }^{240} \mathrm{Pu}(\mathrm{sf})$ data, we compared the data to four different $x$ values between 1.1 and 1.4. The results of the FREYA/TRIPOLI-4 simulations are shown in Fig. 5. Table I lists the values of the neutron multiplicities $\bar{\nu}, \bar{\nu}_{L}$ and $\bar{\nu}_{H}$ for the light and heavy fission fragments. Increasing $x$ for FREYA effectively shifts

TABLE I

AVERAGE NEUTRON MULTIPLICITIES (LIGHT AND HEAVY FISSION FRAGMENTS AND TOTAL) FROM ${ }^{240} \mathrm{PU}(\mathrm{SF})$ AND ${ }^{252} \mathrm{CF}(\mathrm{SF})$. PARAMETERS IN $3^{\text {rd }}$ COLUMN IS $x$ FOR FREYA AND $R_{T}^{\mathrm{MIN}}-R_{T}^{\mathrm{MAX}}{ }_{-} \sigma_{L}-\sigma_{H}$ FOR FIFRELIN.

\begin{tabular}{l|lcrrr}
\hline isotope & model & parameters & $\bar{\nu}_{L}$ & $\bar{\nu}_{H}$ & $\bar{\nu}$ \\
\hline \multirow{3}{*}{${ }^{240} \mathrm{Pu}(\mathrm{sf})$} & & 1.1 & 1.241 & 0.993 & 2.23 \\
& & 1.2 & 1.365 & 0.870 & 2.23 \\
& & 1.3 & 1.487 & 0.748 & 2.23 \\
& & 1.4 & 1.608 & 0.610 & 2.22 \\
\cline { 2 - 6 } & & & & & \\
& & $0.35-1.40-8.5-8.0$ & 1.335 & 0.807 & 2.14 \\
& & $0.5-1.50-8.0-7.5$ & 1.427 & 0.688 & 2.11 \\
& & $0.6-1.70-8.0-7.5$ & 1.552 & 0.556 & 2.11 \\
\hline \multirow{2}{*}{$\mathrm{CIF}(\mathrm{sf})$} & FREYA & 1.27 & 2.18 & 1.58 & 3.76 \\
& FIFRELIN & $0.35-1.40-11-11$ & 2.059 & 1.675 & 3.73 \\
\hline
\end{tabular}

and tilts the correlation from approximately equal intensity at $0^{\circ}$ and $180^{\circ}$ with $x=1.1$ to a significantly higher correlation at $0^{\circ}$ for $x=1.4$. This behavior can be explained by the characteristics of neutron evaporation, as stated earlier. The higher the value of $x$, the more neutrons the light fragment emits. This neutron yield asymmetry leads to a larger correlation at $0^{\circ}$. The neutron correlation measurements determined that $x=1.3$ gives the best match between simulations and data, a value slightly greater than the original one.

Figures 2 and 4 show that for energies below $1200 \mathrm{keV}$, FREYA overpredicts the $180^{\circ}$ correlations. The agreement improves at higher energies. We have adjusted $x$ to the measured ${ }^{240} \mathrm{Pu}(\mathrm{sf})$ neutron-neutron angular correlation data assuming it is single-valued, an assumption common to all isotopes in FREYA. However, comparison with ${ }^{252} \mathrm{Cf}(\mathrm{sf})$ data on the neutron multiplicity as a function of fragment mass suggests

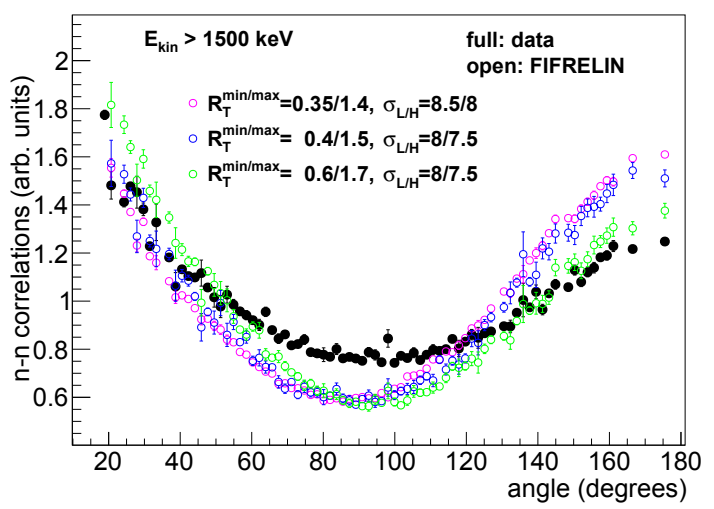

Fig. 6. The cross talk corrected two-neutron angular correlation for ${ }^{240} \mathrm{Pu}(\mathrm{sf})$ as a function of the angular separation and for a neutron kinetic energy threshold of $1500 \mathrm{keV}$. FIFRELIN calculations with different sets of parameters are also shown.

that $x$ should be mass dependent. Adjusting $x$ as a function of fragment mass may improve the overall comparison of the angular correlation data with the simulations.

Compared to FREYA's calculation scheme mentioned above, FIFRELIN does not adjust its free parameters to reproduce the neutron-neutron correlation for ${ }^{252} \mathrm{Cf}(\mathrm{sf})$. The objective of FIFRELIN is to predict fission observables once a fission target observable is achieved. Generally the average prompt neutron multiplicity $\bar{\nu}$ is obtained by adjusting free parameters (temperature ratio law and spin cut-off for primary spin distributions). In the case of ${ }^{252} \mathrm{Cf}(\mathrm{sf})$ the pair $\left(\bar{\nu}_{L}, \bar{\nu}_{H}\right)$ is the target observable and consequently the excitation energy sharing between fragments is more accurately estimated. For instance these average multiplicities are respectively 2.06 and 1.68 for light and heavy fragment groups (compared to reference 2.05 and 1.70). The mean neutron energy spectrum is also well reproduced $(<E\rangle=2.12 \mathrm{MeV}$ in perfect agreement with Mannhart's standard reference [30]). Nevertheless the spectrum shape is not perfect and improvements are still necessary. We have shown that it can be greatly improved [31] by adding $2 \%$ of scission neutrons (emitted at scission before acceleration of fragments). For ${ }^{240} \mathrm{Pu}(\mathrm{sf})$ we have no information on the pair $\left(\bar{\nu}_{L}, \bar{\nu}_{H}\right)$. In such case, FIFRELIN normally defaults to adjusting its four model parameters to reproduce the single $\bar{\nu}=2.14$ value. This is not ideal because of compensation that may arise between light and heavy fragments groups. In this work, in addition to $\bar{\nu}$, angular correlation data can be used to fix the set of model parameters. The effect is shown in Fig. 6. The last set of parameters in Table I was selected to achieve an overall good agreement with measured correlations (Fig.4).

\section{CONCLUSiON}

The event-by-event fission generators FREYA and FIFRELIN have been integrated into and coupled with the Monte Carlo code TRIPOLI-4. The combination of a physics-based fission event generator and an established radiation transport code leads to new capabilities: the simulation of correlations 
that conventional neutron Monte Carlo codes cannot predict. Users can now directly model fissions event-by-event and transport fission secondaries through complex detector geometries while keeping them fully correlated from production to detection.

Two-neutron angular correlation distributions were measured for ${ }^{252} \mathrm{Cf}(\mathrm{sf})$ and ${ }^{240} \mathrm{Pu}(\mathrm{sf})$ using an array of liquid scintillators. The shape of the correlation distributions depends on how the excitation energy is partitioned between the two fission fragments. Experimental data suggest that the lighter fragment is disproportionately excited for spontaneous fission and thermal neutron induced fissions. With the codes TRIPOLI-4 along with FREYA and FIFRELIN, we were able to reproduce the overall shape of the experimentallymeasured neutron-neutron angular correlations. Moreover, for ${ }^{240} \mathrm{Pu}(\mathrm{sf})$, the measured asymmetry was used as a means to adjust the FREYA model parameter $x$, which controls the energy partition between the fragments. It is important to note that this parameter was so far inaccessible in other measurements. Indeed, there are no existing data available to quantify this energy partition for ${ }^{240} \mathrm{Pu}(\mathrm{sf})$. Model parameters in FIFRELIN are not directly linked to the excitation energy sharing but temperature and spin distributions before neutron emission. It is thus more difficult to tune on neutron-neutron correlations. Nevertheless without any information on $\left(\bar{\nu}_{L}, \bar{\nu}_{H}\right)$ the measured neutron-neutron correlations are very useful since they are a signature of the excitation energy sharing and thus of the neutron multiplicity per fragment group. In addition this data can be measured for several neutron kinetic energy thresholds, which enables us to detect potential problems in the simulation of the prompt fission neutron spectrum.

\section{ACKNOWLEDGMENT}

This work was performed under the auspices of the U.S. Department of Energy by Lawrence Livermore National Laboratory under Contract DE-AC52-07NA27344. TRIPOLI-4 ${ }^{\circledR}$ is a registered trademark of CEA. The authors wish to thank Electricité de France (EDF) for partial financial support.

\section{REFERENCES}

[1] J. Randrup and R. Vogt, "Calculation of fission observables through event-by-event simulation," Phys. Rev. C, vol. 80, p. 024601, 2009.

[2] R. Vogt, J. Randrup, J. Pruet, and W. Younes, "Event-by-event study of prompt neutrons from ${ }^{239} \mathrm{Pu}(n, \mathrm{f})$, , Phys. Rev. C, vol. 80, p. 044611 , 2009.

[3] R. Vogt and J. Randrup, "Event-by-event study of neutron observables in spontaneous and thermal fission," Phys. Rev. C, vol. 84, p. 044621, 2011.

[4] R. Vogt, J. Randrup, D. A. Brown, M. A. Descalle, and W. E. Ormand, "Event-by-event evaluation of the prompt fission neutron spectrum from ${ }^{239} \mathrm{Pu}(\mathrm{n}, \mathrm{f}), "$ Phys. Rev. C, vol. 85, p. 024608, 2012.

[5] R. Vogt and J. Randrup, "Event-by-event study of photon observables in spontaneous and thermal fission," Phys. Rev. C, vol. 87, p. 044602 , 2013.

[6] J. Randrup and R. Vogt, "Refined treatment of angular momentum in the event-by-event fission model FREYA," Phys. Rev. C, vol. 89, p. 044601, 2014.

[7] R. Vogt and J. Randrup, "Neutron angular correlations in spontaneous and neutron-induced fission," Phys. Rev. C, vol. 90, p. 064623, 2014.

[8] J. M. Verbeke, J. Randrup, and R. Vogt, "Fission reaction yield algorithm FREYA for event-by-event simulation of fission," Comp. Phys. Comm., vol. 191, pp. 178-202, 2015.
[9] - "Fission reaction yield algorithm FREYA 2.0.2 user manual," LLNL-SM-705798, Lawrence Livermore National Laboratory, submitted to Comp. Phys. Comm.

[10] V. F. Weisskopf, "Statistics and nuclear reactions," Phys. Rev., vol. 52, p. $295,1937$.

[11] J. Terrell, "Fission neutron spectra and nuclear temperatures," Phys. Rev., vol. 113 , p. 527,1959

[12] D. Madland and J. Nix, "New calculation of prompt fission neutron spectra and average prompt neutron multiplicities," Nucl. Sci. Eng., vol. 81, pp. $213-271,1982$.

[13] R. Capote and al., "RIPL - reference input parameter library for calculation of nuclear reactions and nuclear data evaluations," Nucl. Data Sheets, vol. 110, pp. 3107-3214, 2009.

[14] O. Litaize and O. Serot, "Investigation of phenomenological models for the Monte Carlo simulation of the prompt fission neutron and $\gamma$ emission," Phys. Rev. C, vol. 82, no. 5, p. 054616, Nov. 2010. [Online]. Available: http://link.aps.org/doi/10.1103/PhysRevC.82.054616

[15] D. Regnier, O. Litaize, and O. Serot, "Preliminary results of a full Hauser-Feshbach simulation of the prompt neutron and gamma emission from fission fragments," Phys. Procedia, vol. 47, pp. 47 - 52, 2013. [Online]. Available: http://www.sciencedirect.com/science/article/pii/S1875389213004379

[16] —, "An improved numerical method to compute neutron/gamma deexcitation cascades starting from a high spin state," Comp. Phys. Comm., vol. 201, pp. 19 - 28, 2016. [Online]. Available: http://www.sciencedirect.com/science/article/pii/S0010465515004452

[17] O. Litaize, O. Serot, and L. Berge, "Fission modelling with FIFRELIN," Eur. Phys. J. A, vol. 51, no. 12, p. 177, 2015.

[18] N. Varapai, F. J. Hambsch, S. Oberstedt, O. Serot, G. Barreau, N. Kornilov, and S. Zeinalov, "Development of a Digital Technique for the Determination of Fission Fragments and Emitted Prompt Neutron Characteristics," AIP Conf. Proc., vol. 798, pp. 369-372, 2005.

[19] L. Demattè, C. Wagemans, R. Barthélémy, P. D'hondt, and A. Deruytter, "Fragments' mass and energy characteristics in the spontaneous fission of ${ }^{236} \mathrm{Pu},{ }^{238} \mathrm{Pu},{ }^{240} \mathrm{Pu},{ }^{242} \mathrm{Pu}$, and ${ }^{244} \mathrm{Pu}, "$ Nucl. Phys. A, vol. 617, pp. 331-346, 1997.

[20] H. A. Bethe, "An attempt to calculate the number of energy levels of a heavy nucleus," Phys. Rev., vol. 50, pp. 332-341, Aug 1936. [Online]. Available: https://link.aps.org/doi/10.1103/PhysRev.50.332

[21] F. Becvar, "Simulation of $\gamma$ cascades in complex nuclei with emphasis on assessment of uncertainties of cascade-related quantities," Nucl. Instrum. Meth. Phys, Res. A, vol. 417, pp. 434 - 449, 1998. [Online]. Available: http://www.sciencedirect.com/science/article/pii/S0168900298007876

[22] TRIPOLI $4^{\circledR}$ Project Team, "TRIPOLI-4 ${ }^{\circledR}$, CEA, EDF and AREVA reference Monte Carlo code," Ann. Nucl. Energy, vol. 82, pp. 151-160, 2015.

[23] J. M. Verbeke and O. Petit, "Stochastic analog neutron transport with TRIPOLI-4 and FREYA: Bayesian uncertainty quantification for neutron multiplicity counting," Nucl. Sci. Eng., vol. 183, pp. 214-228, 2016.

[24] O. Petit, C. Jouanne, O. Litaize, O. Serot, A. Chebboubi, and Y. Pénéliau, "FIFRELIN-TRIPOLI-4 coupling for Monte Carlo simulations with a fission model. Application to shielding calculations," to be published in Proc. ICRS-13 \& RPSD-2016 (EPJ - Web of Conference).

[25] O. Petit and E. Dumonteil, "Analog neutron transport for nuclear instrumentation applications with the Monte Carlo code TRIPOLI-4," Nucl. Technol., vol. 192, p. 259, 2015.

[26] (2016). [Online]. Available: http://www.eljentechnology.com

[27] J. M. Verbeke, L. F. Nakae, and R. Vogt, "Neutron-neutron angular correlations in spontaneous fission of ${ }^{252} \mathrm{Cf}$ and ${ }^{240} \mathrm{Pu}$," Phys. Rev. C, submitted for publication.

[28] A. M. Gagarski, I. S. Guseva, V. E. Sokolov, G. V. Val'ski, G. A. Petrov, D. O. Krinitsin, D. V. Nikolaev, T. A. Zavarukhina, and V. I. Petrovaal, "Neutron-neutron angular correlations in spontaneous fission of ${ }^{252} \mathrm{Cf}$," Bull. Russ. Acad. Sci.: Phys., vol. 72, p. 773, 2008.

[29] S. A. Pozzi, B. Wieger, A. Enqvist, S. Clarke, M. Flaska, M. Marcath, E. Larsen, R. C. Haight, and E. Padovani, "Correlated neutron emissions from ${ }^{252}$ Cf," Nucl. Sci. Eng., vol. 178, pp. 250-260, 2014.

[30] W. Mannhart, "Evaluation of the ${ }^{252} \mathrm{Cf}$ fission neutron spectrum between $0 \mathrm{Mev}$ and $20 \mathrm{Mev}$," Proc. Advisory Group Mtg. Neutron Sources, Leningrad, USSR, 1986 (IAEA-TECDOC-410), Vienna, 1987.

[31] O. Serot, O. Litaize, and A. Chebboubi, "Influence of scission neutrons on the prompt fission neutron spectrum calculations," Proc. Int. Conf. ND-2016, 11-16 September, 2016, Brugges, Belgium, 2016. 